\title{
Nitrite Improves Heart Regeneration in Zebrafish
}

\author{
Elizabeth R. Rochon, ${ }^{1}$ Maria Azzurra Missinato, ${ }^{2}$ Jianmin Xue, Jesús Tejero,, ${ }^{1,3}$ Michael Tsang, ${ }^{2}$ \\ Mark T. Gladwin, ${ }^{1,3}$ and Paola Corti ${ }^{1,4,5}$
}

\begin{abstract}
Aims: Nitrite is reduced to nitric oxide (NO) under physiological and pathological hypoxic conditions to modulate angiogenesis and improve ischemia-reperfusion injury. Although adult mammals lack the ability to regenerate the heart after injury, this is preserved in neonates and efforts to reactivate this process are of great interest. Unlike mammals, the adult zebrafish maintain the innate ability to regenerate their hearts after injury, providing an important model to study cardiac regeneration. We thus explored the effects of physiological levels of nitrite on cardiac and fin regeneration and downstream cellular and molecular signaling pathways in response to amputation and cryoinjury. Results: Nitrite treatment of zebrafish after ventricular amputation or cryoinjury to the heart in hypoxic water ( $\sim 3$ parts per million of oxygen) increases cardiomyocyte proliferation, improves angiogenesis, and enhances early recruitment of thrombocytes, macrophages, and neutrophils to the injury. When tested in a fin regeneration model, neutrophil recruitment to the injury site was found to be dependent on NO.

Innovation: This is the first study to evaluate effects of physiological levels of nitrite on cardiac regeneration in response to cardiac injury, with the observation that nitrite in water accelerates zebrafish heart regeneration.

Conclusion: Physiological and therapeutic levels of nitrite increase thrombocyte, neutrophil, and macrophage recruitment to the heart after amputation and cryoinjury in zebrafish, resulting in accelerated cardiomyocyte proliferation and angiogenesis. Translation of this finding to mammalian models of injury during early development may provide an opportunity to improve outcomes during intrauterine fetal or neonatal cardiac surgery. Antioxid. Redox Signal. 32, 363-377.
\end{abstract}

Keywords: cardiac regeneration, nitrite, NO, nitric oxide, immune response, cardiomyocyte proliferation

\section{Introduction}

$\mathbf{N}$ ITRITE $\left(\mathrm{NO}_{2}^{-}\right)$IS AN INORGANIC SALT that is present in mammalian tissues at physiologically low concentrations $(1-20 \mu M)(57)$. Nitrite can be reduced to nitric oxide (NO) at low oxygen tensions and low $\mathrm{pH}$ and its bioactivation is mediated by disproportionation or by reduction, catalyzed by redox active heme- or molybdenum-containing proteins $(24,39)$. This pathway is independent of NO synthase enzyme activity and contributes significantly to NO signaling under physiological and pathological hypoxia. In normoxia, nitrite within physiological ranges is considered to be an end product of NO oxidation with limited biological activity.
However, in hypoxia, nitrite represents a circulating source of NO that becomes available by enhanced enzymatic reduction and its utilization is relevant for mammalian vasodilation and metabolism (17).

Administration of nitrite in mice is cytoprotective after ischemia-reperfusion injury in the heart and liver and this protective mechanism is mediated by NO production $(3,12)$. Myoglobin $(\mathrm{Mb})$ is the main reductase enzyme in the heart $(11,19,22,54,62)$. The decrease in oxygen tension switches $\mathrm{Mb}$ from acting as an NO scavenger in normoxia to a nitrite reductase in hypoxia $(22,54,61)$. In addition to $\mathrm{Mb}$, hemoglobin $(\mathrm{Hb})$, neuroglobin, and cytoglobin have all been reported to reduce nitrite to $\mathrm{NO}$ in various mammalian tissues

\footnotetext{
${ }^{1}$ Department of Medicine, Heart, Lung, Blood, and Vascular Medicine Institute, University of Pittsburgh, Pittsburgh, Pennsylvania.

${ }^{2}$ Department of Developmental Biology, University of Pittsburgh, Pittsburgh, Pennsylvania.

${ }^{3}$ Division of Pulmonary, Department of Medicine, Allergy and Critical Care Medicine, University of Pittsburgh, Pittsburgh, Pennsylvania.

${ }^{4}$ Division of Cardiology, Department of Medicine, University of Pittsburgh, Pittsburgh, Pennsylvania.

${ }^{5}$ Ri.MED Foundation, Palermo, Italy.
} 


\section{Innovation}

The role of nitrite in the zebrafish regeneration process is unknown. Our work shows that under hypoxia, physiological levels of nitrite are able to improve the heart's ability to repair after injury in zebrafish amputation and cryoinjury models of heart regeneration. Nitrite enhances regeneration by modulating the immune response through nitric oxide (NO) signaling; faster recruitment of thrombocytes, neutrophils, and macrophages accelerates cardiomyocyte proliferation, angiogenesis, and cardiac repair. Interventions that activate NO signaling pathways via nitrite or other mechanisms may provide an opportunity to improve outcomes in hypoxic conditions such as intrauterine fetal or neonatal cardiac surgery.

during hypoxia with significant physiological effects, such as vasodilation and cardioprotection through modulation of mitochondrial respiration $(7,24,26,40,55,68)$.

Measurements of NO metabolites such as nitrite and nitrate in fish blood reveal levels comparable to mammals and exposure to nitrite significantly increases NO production $(20,27)$. We found that nitrite can be reduced to NO in zebrafish blood by Globin X, a newly discovered erythrocyte globin possibly representing an important ancient role for globins in concert with nitrite (8).

Zebrafish (Danio rerio) is a hypoxia semi-tolerant species similar to its goldfish (Carassius auratus) and carp (Carassius carassius) relatives. They are capable of surviving and being fully functional at very low levels of environmental oxygen $(20,59,72)$. Exposure to hypoxia in goldfish causes large decreases in plasma nitrite, suggesting its transfer or utilization (20). The crucian carp have developed unique strategies to survive hypoxia and it maintains normal cardiac output during anoxia-exposed periods. In these conditions, high levels of endogenous nitrite accumulate in the anoxic heart and during anoxia/reoxygenation, nitrite is reduced to NO, protecting the cardiac muscle from ischemic injury (59). Nitrite is known to be toxic at high concentrations in fish but the nitrite effect at physiological concentrations appears to be protective in the heart $(37,74)$. Importantly, the effects of nitrite on cardiac regeneration have not been studied.

As opposed to adult mammals, the zebrafish have the ability to regenerate cardiac muscle after surgical amputation or damage to a portion of the ventricle $(4,36,52)$. The mechanisms that regulate the regenerative ability in the early stages of mammalian development are subject to intense research due to its therapeutic potential for treating heart disease (2). Despite the differences with the mammalian heart, the mechanisms underpinning cardiac regeneration are conserved in fish and mammals (70). After injury, there is an initial inflammatory response that initiates the regenerative program to build new tissue involving proliferation of cardiomyocytes, and neovascularization of the injured area through angiogenesis (2). The importance of an early immune response in the regenerative process has been recognized but much remains to be explored $(1,13,16,38,65)$.

Here, we demonstrate that nitrite treatment in hypoxia improves cardiac regeneration in zebrafish by altering the immune system response, characterized by early recruitment of thrombocytes, neutrophils, and macrophages. The consequent improvement on the healing process and clearance of the scar leads to beneficial effects on the injury size, cardiomyocyte proliferation, and neovascularization. We also examined the nitrite effect on the neutrophil response in a larval fin regeneration model and found that increased neutrophil recruitment was dependent on NO production. This study provides insights for the use of nitrite not only as a therapeutic drug against reperfusion damage of ischemic hearts but also as an enhancer of regeneration that can potentially be induced in mammals at exposure concentrations commonly found in nature.

\section{Results}

\section{Treatment with nitrite in a hypoxic environment increases the rate of cardiac regeneration after amputation or cryoinjury}

Unlike mammals, fish are often exposed to nitrite in water. Under normoxic environmental conditions, physiological concentrations of nitrite are not detrimental (37). Although toxic levels have been studied extensively, there has been a lack of evaluation on physiological levels of nitrite exposure. This is particularly interesting because under low oxygen conditions, nitrite can be reduced to NO through the action of heme-containing proteins and small amounts of nitrite can initiate NO-based signaling with consequent physiological effects (17). To determine whether nitrite treatment has an effect on cardiac regeneration in zebrafish, we created a model where fish were treated with nitrite in normoxic or hypoxic conditions and cardiac regeneration was assessed in both an amputation and cryoinjury model.

Nitrite levels were determined in the water used to house zebrafish using an NO ozone-based chemiluminescent analyzer (nitric oxide analyzer [NOA]) and found to be $0.14 \mu \mathrm{M}$ with zebrafish cardiac tissue levels being between $2.82 \pm 1.32 \mu M$ and $3.69 \pm 0.43 \mu M$, with no difference observed before or after injury (Fig. 1A). Zebrafish were exposed to a hypoxic environment by bubbling $5 \%$ oxygen balanced nitrogen into the water, dropping oxygen concentrations in the water from $\sim 7.0$ to $\sim 2.5 \mathrm{~g} / \mathrm{mL}$. Tissue hypoxia was confirmed by using the previously validated hypoxyprobe staining (29), showing a significant increase in tissue fluorescence intensity upon hypoxia exposure compared with normoxia, with the epicardium being the most hypoxic region (Fig. 1D, E).

To determine the nitrite dose, fish were treated with 10,30, and $60 \mu \mathrm{m}$ sodium nitrite in hypoxic water and preliminary effects on cardiac regeneration were queried, including size of injury (Fig. 1F) and cardiomyocyte proliferation after cryoinjury. At $10 \mu M$ nitrite, there was both a significant decrease in the injury size and an increase in the number of proliferating cardiomyocytes measured at 5 days postcryoinjury (5 dpc) (Fig. 1B, C). Although both 30 and $60 \mu \mathrm{m}$ sodium nitrite did not have a detrimental effect on cardiac healing, they also did not have a positive effect on both scar size and proliferation (Fig. 1B, C). For this reason, subsequent experiments were performed with $10 \mu M$ nitrite.

We investigated the extent of regeneration after cardiac injury with a full factorial analysis of normoxia and hypoxia in the presence or absence of nitrite and looking at both models of heart regeneration: amputation and cryoinjury at 5 and 10 days after injury. The injured region of the heart was visualized with Acid Fuchsin Orange G (AFOG) staining to label the healthy uninjured myocardium in orange, and the injured region in blue (collagen) and red (fibrin). 

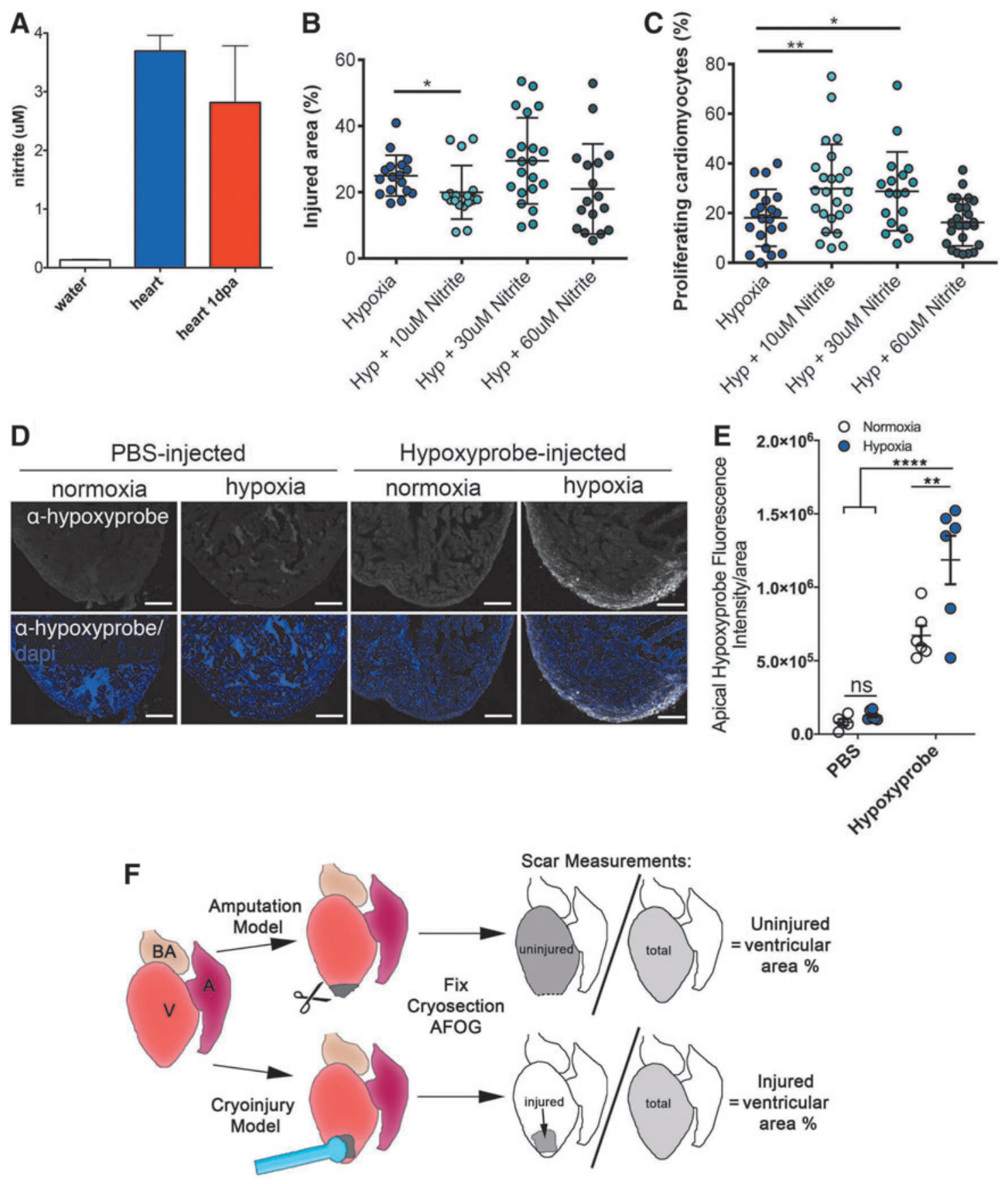

FIG. 1. Nitrite dose-response effect on scar size and cardiomyocyte proliferation after injury. (A) Chemiluminescence detection of nitrite levels in fish water and cardiac tissues measured by using a NOA. Although there is almost no detectable nitrite in the water $(0.14 \mu M)$, the endogenous nitrite concentration in the uninjured heart and in the amputated heart is $3.69 \pm 0.43 \mu M$ and $2.82 \pm 1.32 \mu M$, respectively $(n=3-4)$. After cryoinjury, the injury size as percent of total ventricular area (B) and the number of proliferating cardiomyocytes as percent of total proliferating cells $(\mathbf{C})$ were measured by comparing hypoxia (hyp), hyp $+10 \mu M$ nitrite, hyp $+30 \mu M$ nitrite, and hyp $+60 \mu M$ nitrite. The $10 \mu M$ nitrite dose both significantly decreased the scar size and increased the number of proliferating cardiomyocytes at $5 \mathrm{dpc}$. Student's $t$-test: $* p<0.05, * * p<0.01$. Data are expressed as mean \pm SEM. (D) Hypoxyprobe detection in uninjured wild type hearts injected with either $20 \mu \mathrm{L}$ PBS or $5 \mathrm{mg} / \mathrm{mL}$ hypoxyprobe for 3 days after exposure to normoxia or hypoxia. Hearts were co-stained for DAPI $(n=5-7)$. Scale bar $=100 \mu \mathrm{m}$. (E) Quantification of fluorescence intensity of the ventricle apex. One-way ANOVA $(* * p<0.01 ; * * * * p<0.000)$ with Tukey's multiple-comparison test. Data are expressed as mean \pm SEM. (F) Schematic of amputation and cryoinjury heart regeneration models. After fixation, the hearts were cryosectioned and histologically stained to measure injury sizes. In amputated hearts, the total uninjured area over the total ventricular area was calculated. In cryoinjured hearts, the total injured area over the total ventricular area was calculated. A, atrium; ANOVA, analysis of variance; BA, bulbous arteriosus; dpc, days post-cryoinjury; NOA, nitric oxide analyzer; ns, no significance; PBS, phosphate buffered saline; SEM, standard error of the mean; V, ventricle. 
To assess the injury in the amputation model, we measured the uninjured region of the ventricle because it was not possible to accurately determine the area of the amputated portion after its removal from each heart (Fig. 1F). Although no difference was observed between the normoxia, normoxia/nitrite, and hypoxia, hypoxia/nitrite treatment increased the total percentage of uninjured ventricle area at 5 days post-amputation (dpa) compared with the normoxic and hypoxic conditions (Fig. 2A, B). This difference was no longer visible at $10 \mathrm{dpa}$ (Fig. 2B). Similar to the amputation model, we find that there is an improvement in regeneration at $5 \mathrm{dpc}$ in the hypoxia/nitrite-treated hearts compared with the normoxic and hypoxic hearts where the size of the injured region was measured directly (Fig. 2C, D). This effect is seen only at low concentrations of nitrite $(10 \mu M)$ (Fig. 1B).

At $10 \mathrm{dpc}$, hypoxia/nitrite treatment continues to improve the size of the injury compared with normoxia and normoxia/nitrite treatments but the injured area is similar to hypoxia only, suggesting that hypoxia alone also improves injury (Fig. 2D). These studies suggest that nitrite treatment in a hypoxic environment improves heart regeneration at early stages of regeneration in two zebrafish models of cardiac injury.

\section{Treatment with hypoxia/nitrite enhances early cardiomyocyte proliferation during regeneration}

Proliferation of cardiomyocytes is a central process in cardiac regeneration $(28,32,80)$. To determine whether improved regeneration is potentially driven by increased cardiomyocyte proliferation, hearts were co-stained with anti-proliferating cell nuclear antigen (Pcna) and myocyte enhancer factor 2c (Mef2C) to specifically label mitotic cardiomyocyte nuclei. There is a small but significant increase in the number of proliferating cardiomyocytes at $5 \mathrm{dpa}$ when comparing the hypoxia/nitrite-treated hearts with the hypoxia-only hearts, with a trend toward improved proliferation compared with normoxic hearts (Fig. 3A, B). This effect is no longer significant at 10 dpa (Fig. 3B).

The effect of hypoxia/nitrite is more robust in the cryoinjury model at $5 \mathrm{dpc}$, with a significant increase in cardiomyocyte proliferation observed in the hypoxia/nitrite-treated hearts compared with the normoxia or the hypoxia-alone hearts (Fig. 3C, D). Although there is a trend toward increased cardiomyocyte proliferation at $10 \mathrm{dpc}$ in the hypoxia/ nitrite-treated hearts, it is not significant (Fig. 3D). It should
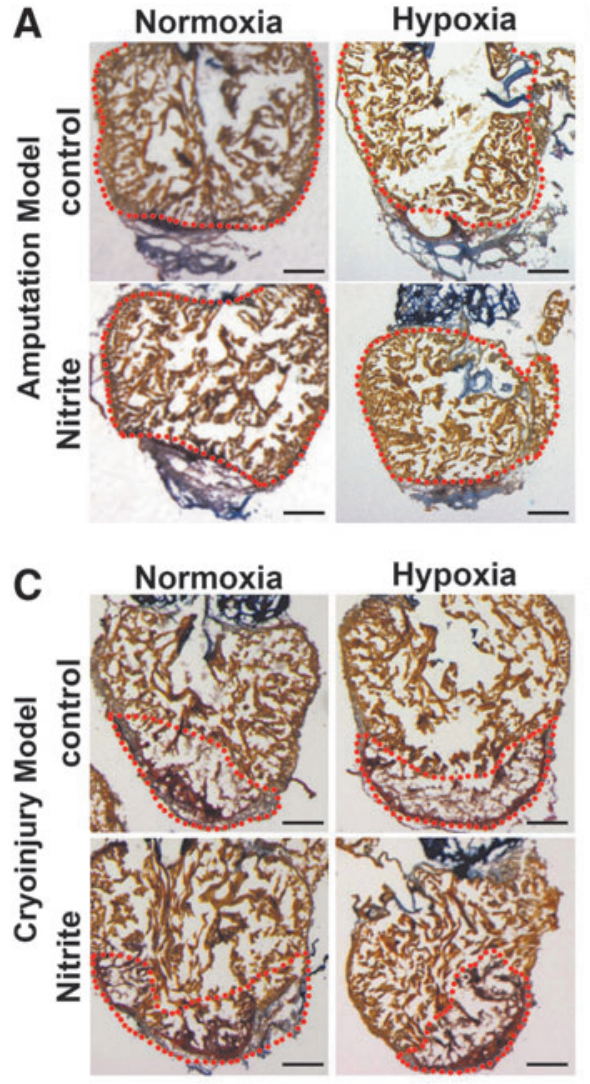
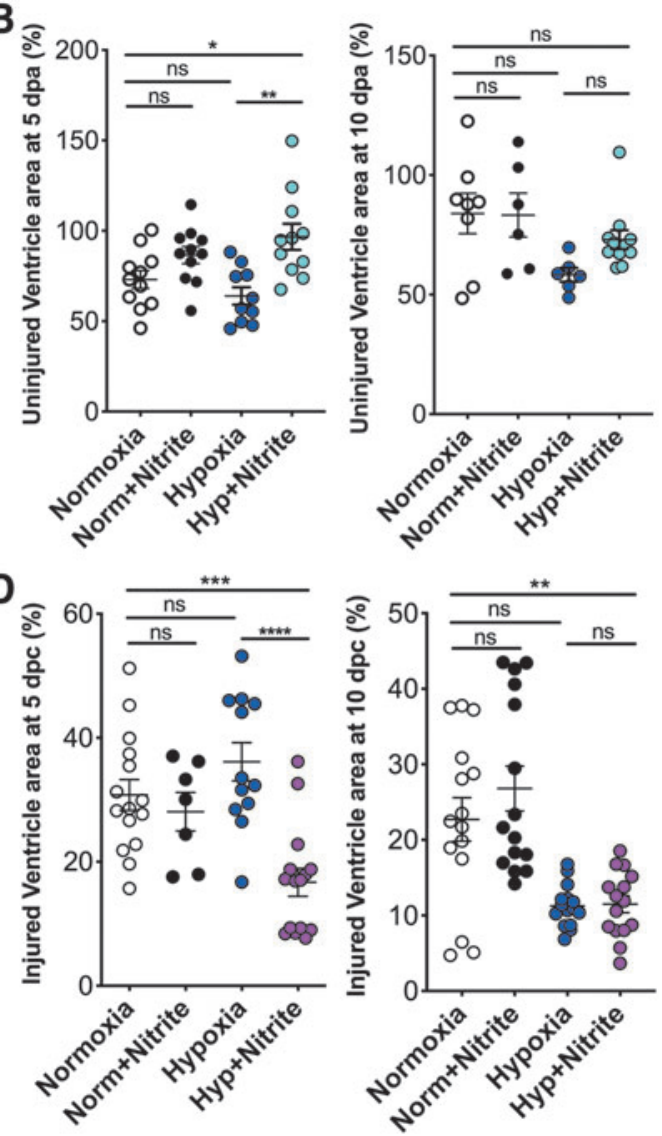

FIG. 2. Nitrite treatment in hypoxia decreases the injury size early in the regenerative process. (A) Representative images of cryosectioned hearts at 5 dpa stained with AFOG, labeling the myocardium in orange, collagen in blue, and fibrin in red. The uninjured myocardium used for quantification is outlined in red. Scale bar $=250 \mu \mathrm{m}$. (B) Quantification of the uninjured area in amputated hearts normalized by total ventricle area at 5 dpa (left) and 10 dpa $($ right). $* p<0.05, * * p<0.01$. Data are expressed as mean \pm SEM $(n=6-16)$. (C) Representative images of cryosectioned hearts at 5 dpc stained with AFOG. The injury is outlined in red. Scale bar $=250 \mu \mathrm{m}$. (D) Quantification of the injured area in cyroinjured hearts normalized by total ventricle area at $5 \mathrm{dpc}($ left $)$ and $10 \mathrm{dpc}($ right). One-way ANOVA $(* * * * p<0.0001)$ with Tukey's multiple-comparison test; $* * p<0.01, * * * p<0.001$, ****p $<0.0001$; ns, no significance. Data are expressed as mean \pm SEM $(n=6-16)$. AFOG, Acid Fuchsin Orange G; dpa, days post-amputation. 

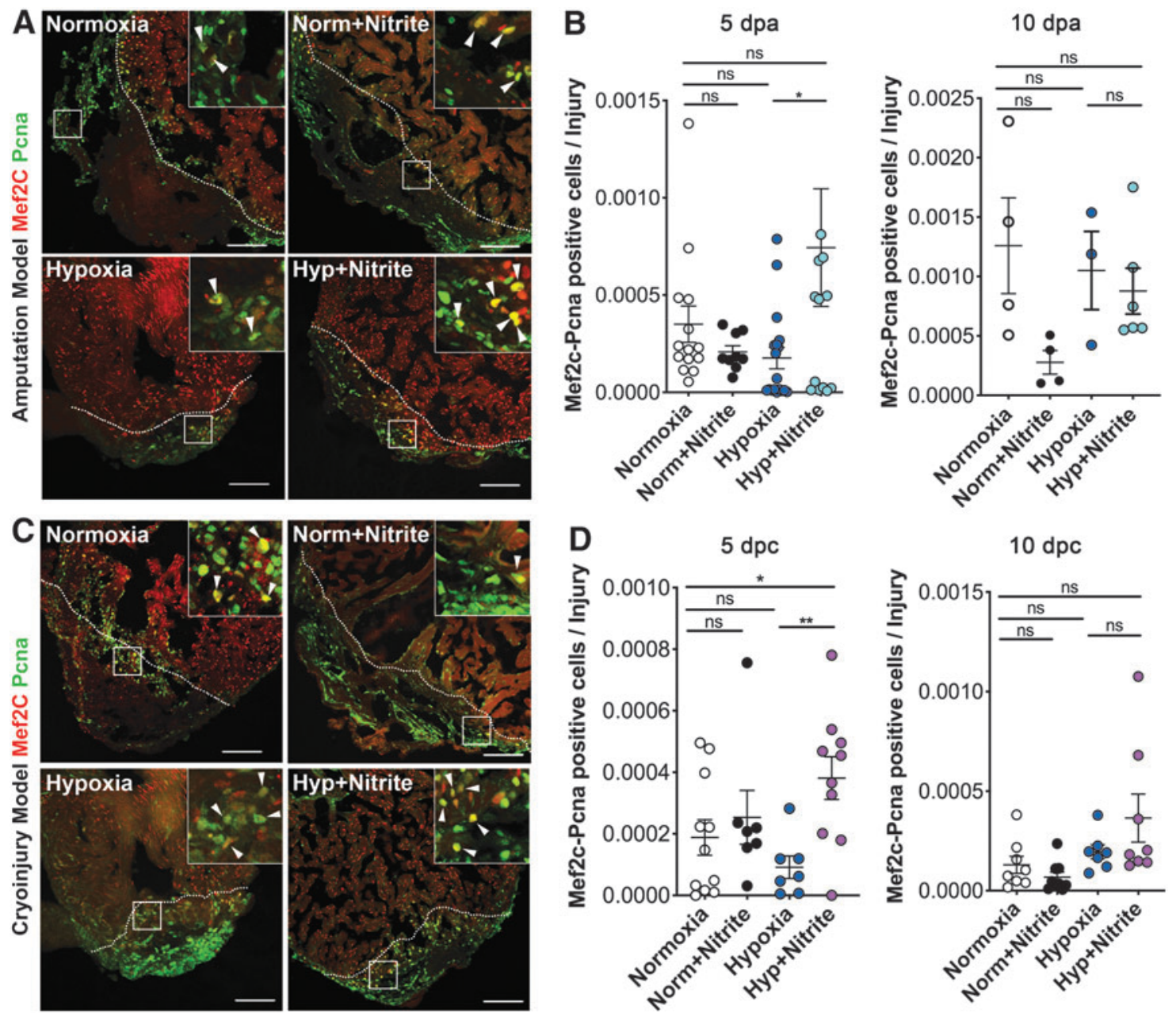

FIG. 3. Treatment with hypoxia/nitrite improves early cardiomyocyte proliferation during regeneration. (A) Immunostaining of amputated hearts with anti-Pcna antibody (green) and anti-Mef2C antibody to label cardiomyocytes (red). Insets indicate a high-magnification view of the regenerating area. Images are $2 \mathrm{D}$ confocal projections. Dashed lines delineate the injury, and white arrowheads point to selected double-positive nuclei. Scale bar $=100 \mu \mathrm{m}$. (B) Quantification of Mef2C/Pcnapositive cells at the injury site in amputated hearts at $5 \mathrm{dpa}$ (left) and $10 \mathrm{dpa}$ (right). One-way ANOVA significance is $p<0.001$; ${ }^{*} p<0.05$. Data are expressed as mean $\pm \operatorname{SEM}(n=3-14)$. (C) Immunostaining of cryoinjured hearts with anti-Pcna (green) and anti$\mathrm{Mef} 2 \mathrm{C}(\mathrm{red})$. Insets indicate a high-magnification view of the regenerating area. Images are 2D confocal projections. Dashed lines delineate the injury, and white arrowheads point to selected double-positive nuclei. Scale bar $=100 \mu \mathrm{m}$. (D) Quantification of Mef2C/ Pcna positive cells at the injury in cryoinjured hearts at $5 \mathrm{dpc}($ left $)$ and $10 \mathrm{dpc}($ right). One-way ANOVA significance is $p<0.05$ at 5 and $10 \mathrm{dpc}$. Tukey's multiple-comparison test; * $p<0.05$, $* * p<0.01$. Data are expressed as mean \pm SEM $(n=7-12)$. 2D, twodimensional; mef2C, myocyte enhancer factor $2 \mathrm{c}$; Pcna, proliferating cell nuclear antigen.

be noted that hypoxia treatment alone in the context of an uninjured heart also had no effect on cardiomyocyte proliferation, with no difference in the number of proliferating cardiomyocytes when compared with normoxic uninjured hearts (Supplementary Fig. S1A, B). We found a small number of proliferating cardiomyocytes in the uninjured hearts, in line with previous observations that there is only a low level of proliferating cardiomyocytes in the absence of injury in the adult zebrafish heart (52).

\section{Hypoxia/nitrite treatment improves neovascularization of the injury}

Efficient neovascularization is necessary for zebrafish cardiac regeneration and has an impact on cardiomyocyte proliferation (46). Importantly, nitrite reduction to NO has been shown to accelerate angiogenesis in mammalian models of peripheral vascular disease and vaso-occlusion (34). In our study, the effects of nitrite are only observed in hypoxia in the regenerating hearts, suggesting that nitrite reduction to $\mathrm{NO}$ may be the main mechanism through which nitrite is exerting its proregenerative effect.

To determine whether nitrite treatment affects neovascularization during regeneration, we examined blood vessel formation in the injured area after amputation by using the $T g(f l i 1: n E G F P)^{y 7}$ line, which labels endothelial cells with GFP and quantified fluorescence intensity normalized to the injured area. Although the hypoxia treatment showed a trend for increased vascular endothelial cells within the injured area compared with normoxia and normoxia/nitrite treatments, there was a significant increase in the hypoxia/nitritetreated group (Fig. 4A, B). The increase between hypoxia and 

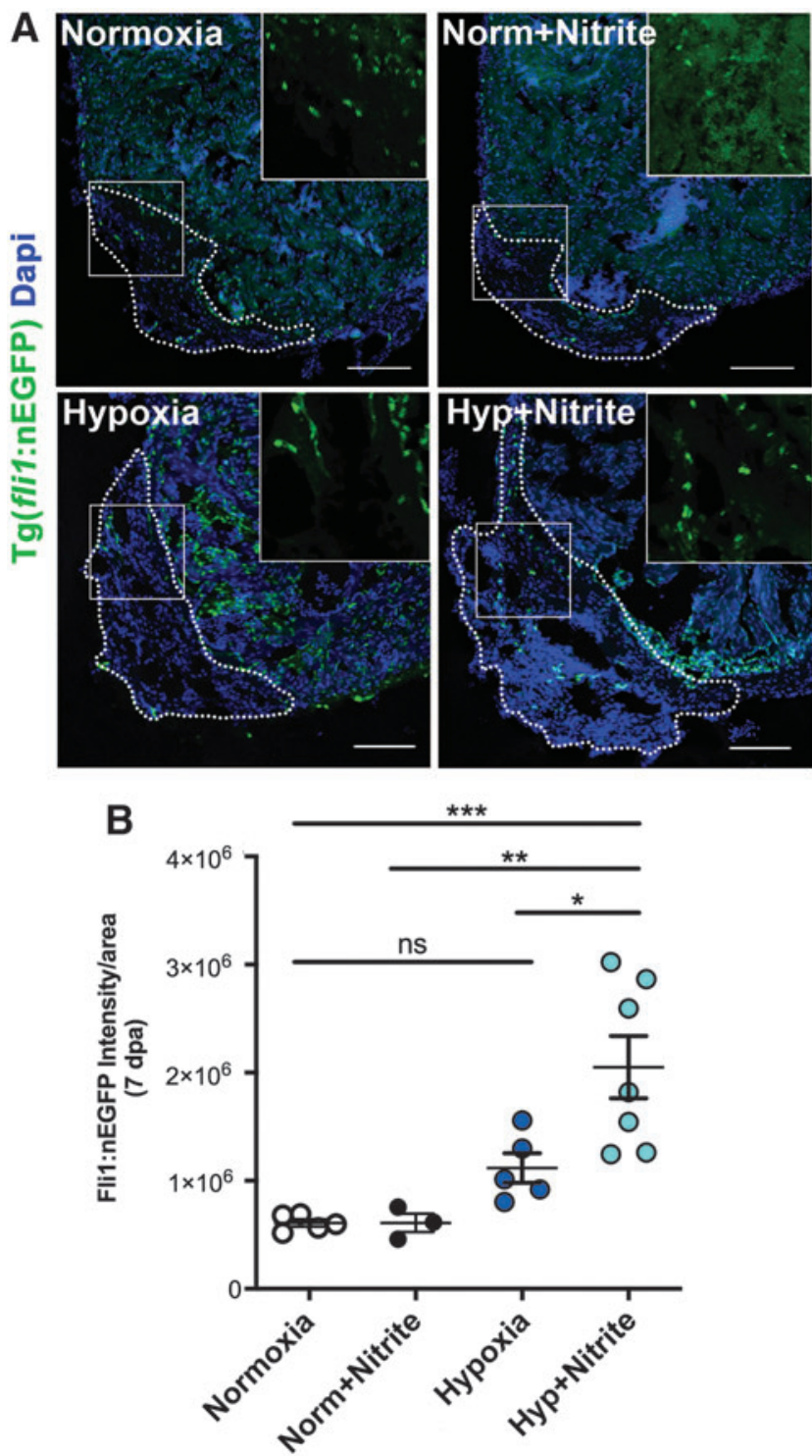

FIG. 4. Nitrite treatment in hypoxia improves neovascularization of the injured area. (A) Analysis of $T g(f l i 1: n E G F P)^{y 7}$ amputated hearts labeling endothelial cells nuclei in green and DAPI in blue after treatment with or without nitrite in normoxia and hypoxia. Images are 2D confocal projections, and insets indicate a high-magnification view of the injured area. Scale Bar: $100 \mu \mathrm{m}$. (B) Quantification of the mean fluorescence intensity normalized to injured area at $7 \mathrm{dpa}(n=$ $3-7)$. One-way ANOVA $(* * * p<0.001)$ with Tukey's multiplecomparison test; $* p<0.05, * * p<0.01, * * * p<0.001$. Data are expressed as mean \pm SEM. Dashed lines delineate the injury.

hypoxia/nitrite is also observed in the cryoinjury model at 5 dpc (Supplementary Fig. S2A, B). These results suggest that treatment with hypoxia/nitrite improves angiogenic sprouting in the injured area and may support cardiomyocyte proliferation and decreased injury size.

\section{Hypoxia/nitrite increases thrombocyte recruitment to the site of cardiac injury and repair}

Initial inflammatory responses after injury set the stage for the cardiac regenerative program to resolve toward generation of new cardiac tissue rather than fibrosis. Nitrite is shown to have an anticoagulation effect on human platelets in the presence of fish red blood cells (8). Although zebrafish lack platelets, they possess their functional equivalent, thrombocytes. Thrombocytes play important roles in vascular hemostasis by forming the initial plug that prevents bleeding and similar to their platelet equivalents, can modulate inflammation, angiogenesis, and tissue repair $(31,51)$.

$\operatorname{Tg}(c d 41-G F P)^{l a 2}$ fish selectively labels thrombocytes in green (45). The effect of nitrite in normoxia and hypoxia on thrombocytes at 1 dpa was evaluated. $T g(c d 41-G F P)^{l a 2}$ hearts were immunostained with anti-sarcomeric actin to label uninjured/fully differentiated cardiomyocytes red, leaving the injured area labeled with DAPI alone (Fig. 5). We find a significant increase in thrombocytes in the injury after hypoxia/ nitrite treatment compared with hypoxia alone and these results also show an increasing trend when compared with normoxia conditions. (Fig. 5). These data suggest that hypoxia/nitrite treatment potentiates the initial immune response by increased thrombocyte accumulation 1 day after injury.

\section{Hypoxia/nitrite increases macrophage and neutrophil recruitment to the site of cardiac injury and increases clearance of erythrocytes}

Inhibition of the inflammatory response in adult zebrafish altering the infiltration of neutrophils and macrophages in the injured myocardium leads to impaired cardiac repair $(10,23$, 38). Macrophages are key immune cells that function to remove dead cell and debris after injury. Efficient recruitment of macrophages has been demonstrated to be essential for cardiac regeneration in the neonatal mouse and zebrafish $(1,10)$. Using the transgenic line, Tg(mpeg1:dendra2 $)^{u w m 12}$, which specifically labels macrophages (77), we counted mpeg 1-positive cells in the injury after amputation. We found that in the presence of hypoxia/nitrite, there is a small but significant increase in the number of macrophages recruited to the wounded area at 7 dpa compared with hypoxia treatment alone (Fig. 6A, B), with no significant differences compared with normoxia or hypoxia treatments.

Neutrophil recruitment to the site of injury has also been demonstrated to facilitate regeneration after ventricular amputation $(24,38)$. Neutrophils are the first leukocytes to be recruited to sites of inflammation and contribute to the removal of dead cell and matrix debris [reviewed in Chen and Frangogiannis (5)]. After a careful analysis of the dynamics of neutrophil recruitment in the injured heart (Supplementary Fig. S3) by using the $T g(l y z: e G F P)^{k o 02}$ line that specifically labels neutrophils in green (51), we analyzed the effects of nitrite in normoxia and hypoxia on the number of neutrophils recruited to the injury site after amputation at the peak recruitment phase of $3 \mathrm{dpa}$.

Interestingly, we observed a trend toward a decrease in the number of neutrophils recruited at $3 \mathrm{dpa}$ in the hypoxic-treated hearts compared with normoxia and normoxia/nitrite (Fig. 7A, C). Treatment with nitrite in hypoxia was able to increase the number of neutrophils in the injured area, resembling normoxic levels, suggesting an interaction between hypoxia and nitrite that affects neutrophil recruitment and/or behavior (twoway analysis of variance [ANOVA], $p=0.0353$ ). No significant difference was observed in neutrophil number at the later 

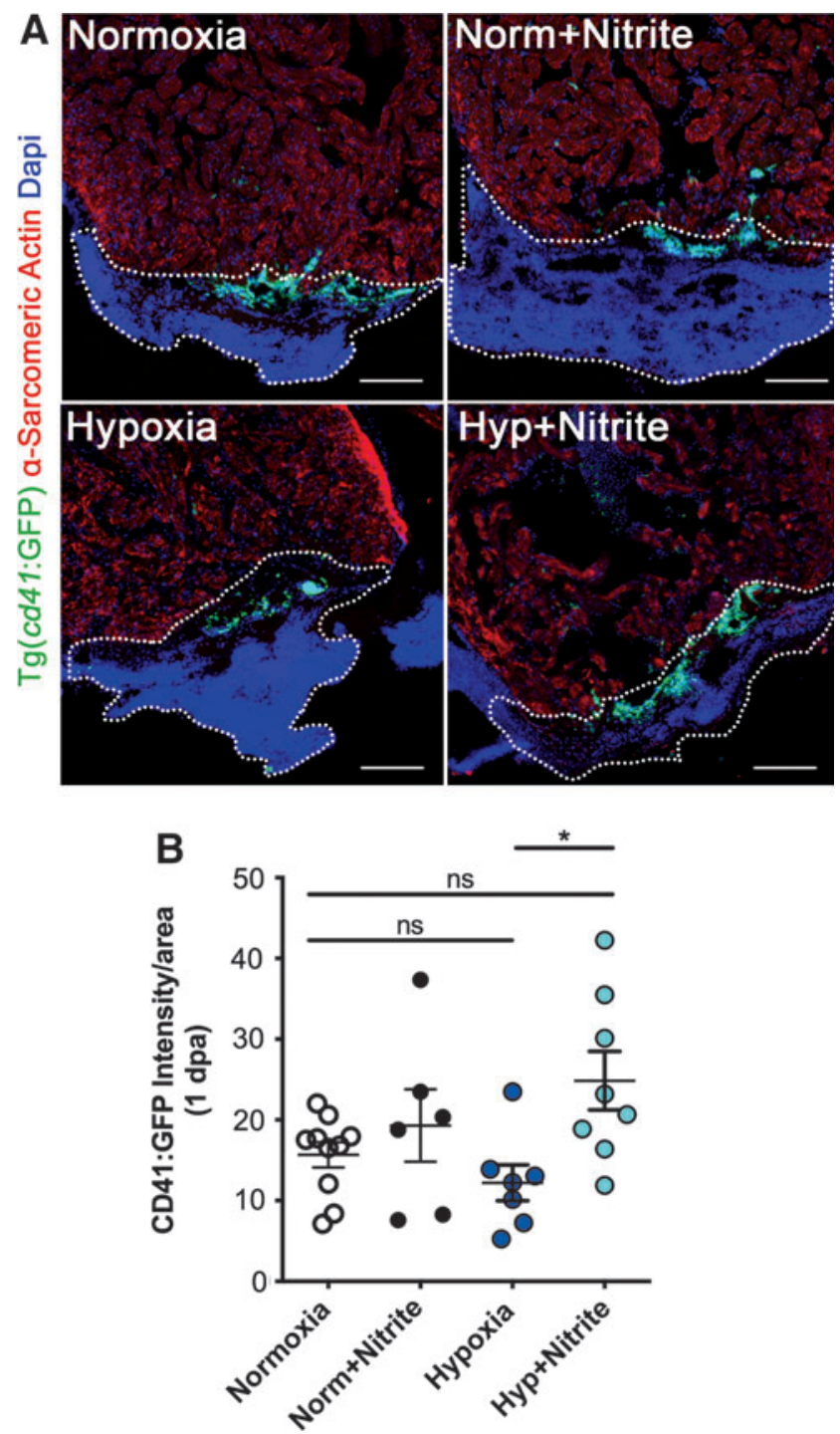

FIG. 5. Thrombocyte accumulation is increased by hypoxia/nitrite treatment. (A) Confocal images of Tg(cd41:GFP $)^{l a 2}$ hearts labeling thrombocytes in green, antisarcomeric actin in red, and DAPI in blue with or without nitrite in normoxia and hypoxia. Injured area is outlined with a white dashed line. Images are 2D confocal projections. Scale Bar: $100 \mu \mathrm{m}$. (B) Quantification of GFP fluorescence intensity normalized to injury area at $1 \mathrm{dpa}(n=6-8)$. Oneway ANOVA $(* p<0.05)$ with Tukey's multiple-comparison test; $* p<0.05$. Data are expressed as mean \pm SEM.

timepoint of 5 dpa (Fig. 7B, D). This analysis performed in the cryoinjury model confirms a significant increase in Lyzpositive cells in the hypoxia nitrite-treated hearts compared with hypoxia at $5 \mathrm{dpc}$ (Supplementary Fig. S2C, D).

Since macrophages and neutrophils function to remove dead cells and debris within the injured area, we next evaluated the clearance of erythrocytes from the initial clot that is formed after injury on treatment with nitrite. To measure this, we analyzed the fluorescence intensity of the injured area of $T g(\text { gatal:DsRed })^{s d 2}$, expressing DsRed in the erythrocytes. We found that indeed, on treatment with hypoxia/nitrite there is a significant decrease in DsRed
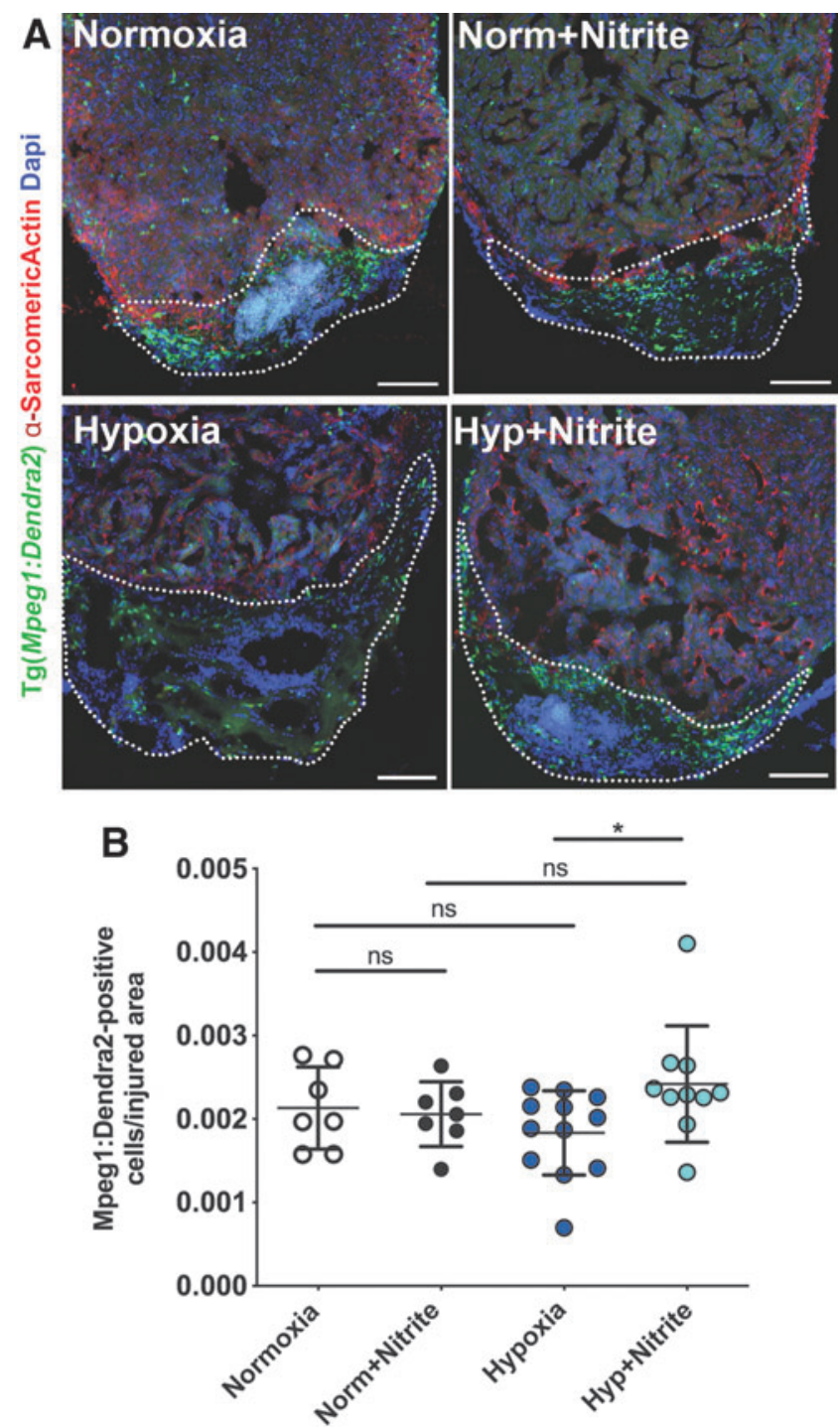

FIG. 6. Nitrite treatment in hypoxia increases the number of macrophages at the site of injury. (A) Analysis of $T g$ (mpeg1:dendra2) ${ }^{u w m 12}$ stained with anti-sarcomeric actin in red and DAPI in blue 7 days after amputation with or without nitrite in normoxia and hypoxia. Injured area is outlined with a dashed line. Images are 2D confocal projections $(n=7-12)$. Scale Bar: $100 \mu \mathrm{m}$. (B) Quantification of Mpeg1-positive cells within the injured area at $7 \mathrm{dpa}$. Student's $t$-test comparing hypoxia and hypoxia/nitrite treatment: ${ }^{*} p<0.05$. Data are expressed as mean \pm SEM.

fluorescence intensity at 3 dpa compared with normoxia and hypoxia treatments (Fig. 7A, C).

At $5 \mathrm{dpa}$, the amount of erythrocytes in the injury is similar in the presence or absence of nitrite in both normoxia and hypoxia (Fig. 7B, D), suggesting that the clearance of the red blood cell clot is accelerated early on by hypoxia/nitrite treatment and correlates with the concomitant increase in neutrophils. Similarly, in the cryoinjury model, we see a significant decrease in the erythrocyte fluorescence intensity at $5 \mathrm{dpc}$ in the hypoxia/nitrite-treated hearts compared with hypoxia alone, also correlating with increased neutrophils during this time (Supplementary Fig. S2E, F). 
A
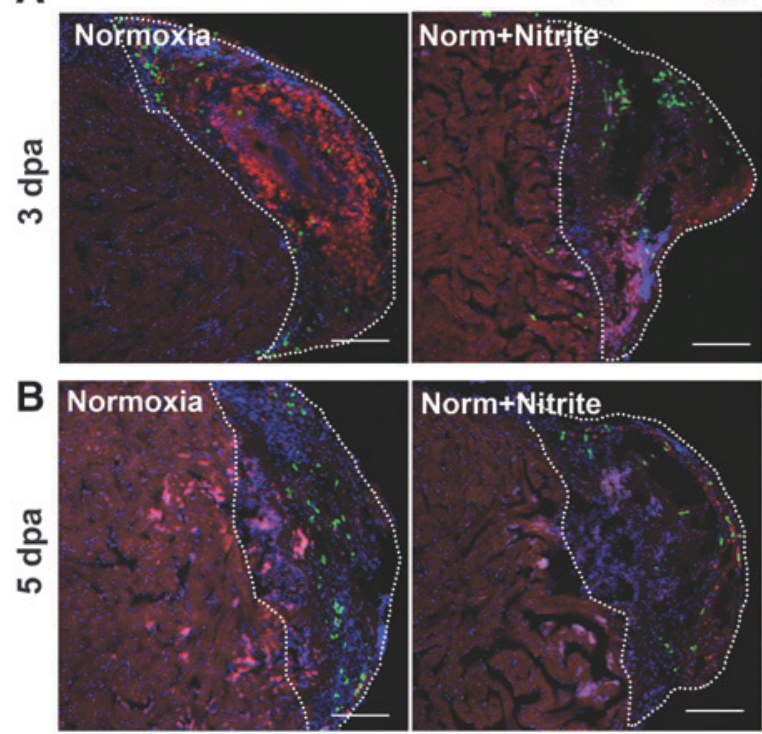

C

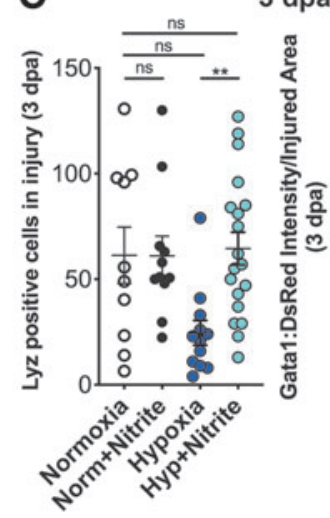

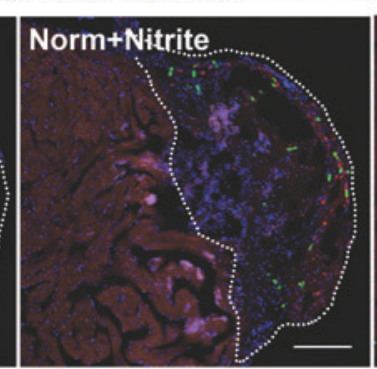

D

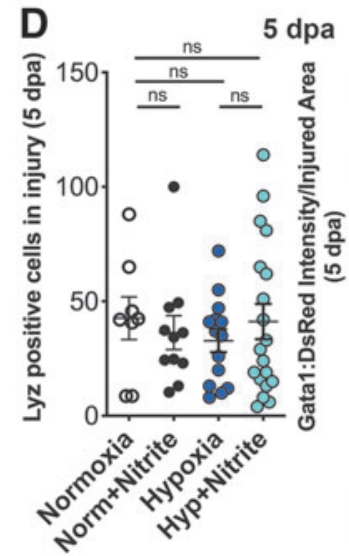

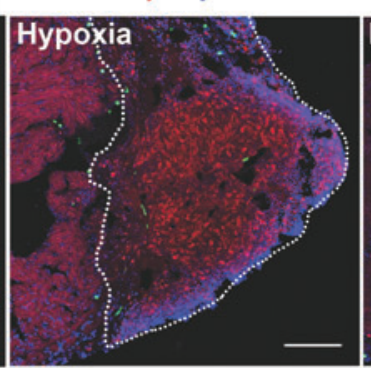
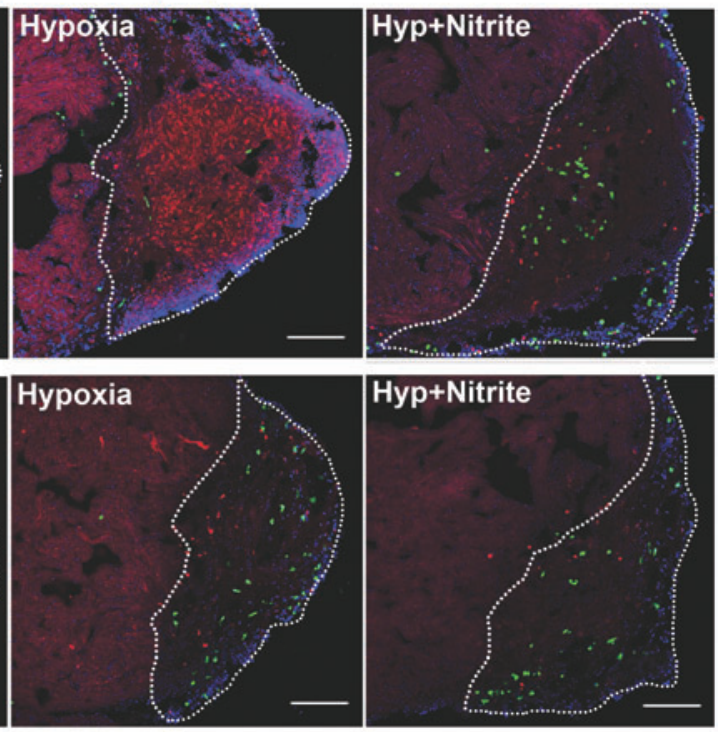

E

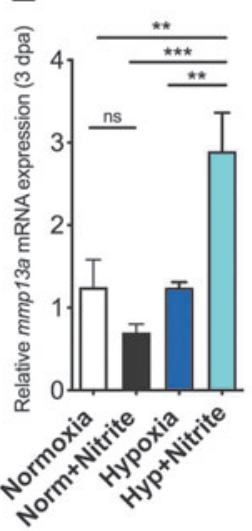

FIG. 7. Nitrite treatment in hypoxia increases the number of neutrophils and decreases the number of erythrocytes at the site of injury in an amputation model. Confocal 2D maximum projections of $T g(\text { lyz:GFP })^{k 0 O} ;(\text { gatal:Dsred })^{s d 2}$ hearts, labeling neutrophils in green and erythrocytes in red (with dapi in blue) in amputated hearts at 3 dpa (A) and 5 dpa (B) after treatment with or without nitrite in normoxia or hypoxia. The injured area is outlined with white dashed lines. Images are 2D confocal projections. Scale Bar: $100 \mu \mathrm{m}$. (C) Quantification of Lyz-positive cells (one-way ANOVA, ** $p<0.01$ ) within the injured area and quantification of Gata1 fluorescence intensity normalized by injury area at 3 dpa $(n=8-19)$. (D) Quantification of Lyz-positive cells in the injured area and quantification of Gata1 fluorescence intensity normalized by injury area at 5 dpa $(n=8-19)$. One-way ANOVA with Tukey's multiple-comparison test; $*^{*}<0.05$, ** $p<0.01$. Data are expressed as mean \pm SEM. (E) Expression levels of mmp13a normalized to polr $2 d$ in 3 dpa hearts treated with or without nitrite in normoxia or hypoxia. Samples values are fold change compared with normoxic 3 dpa hearts ( $n=5$ per treatment, each data point is a pool of eight hearts). One-way ANOVA $(* * * p<0.001)$ with Tukey's multiplecomparison test; $* * p<0.01$ and $* * * p<0.001$.

\section{Expression of proinflammatory mmp13a is increased by hypoxia/nitrite treatment in the heart after injury}

Macrophage-related processes are associated with their ability to migrate throughout the body and remodel surrounding stroma by degrading the extracellular matrix via secretion of matrix metalloproteinases (MMPs) (73). MMPs play important roles in tissue remodeling and MMP9 and 13a are expressed by myeloid cells in both human and zebrafish $(53,78)$. Recently, a positive role for MMPs in neutrophil and macrophage recruitment during early phases of cardiac regeneration in zebrafish has been revealed (76). With this in mind, we assayed the expression level by quantitative reverse transcription polymerase chain reaction (qRT-PCR) of mmpl3a in normoxia and hypoxia with and without nitrite at $3 \mathrm{dpa}$, the time when we see the highest recruitment of neutrophils to the injured area. Interestingly, we find that hypoxia/nitrite treatment significantly increases the expression of mmpl3a compared with normoxia, normoxia/nitrite, and hypoxia treatments (Fig. 7E).

Changes observed in mmp13a are only observed on treatment with hypoxia/nitrite, and not under normoxia/nitrite or hypoxia, suggesting an interaction between hypoxia and nitrite, confirmed by analysis by two-way ANOVA $(p=0.0017)$. These data provide evidence that hypoxia/nitrite treatment during cardiac regeneration initiates a transcriptional response in the heart that favors an increased immune response via mmpl3a. 


\section{Hypoxia/nitrite treatment alters neutrophil recruitment in a fin regeneration model}

The interaction between hypoxia and nitrite treatment suggests that nitrite may be reduced to NO to exert its effects. To determine whether the effects of hypoxia and nitrite treatment on immune cell recruitment are dependent on NO formation, we used a larval fin regeneration model. Larval models allow for manipulation of molecular pathways by adding small molecules and drug treatments directly to the water, where they are able to diffuse into the animal $(48,50)$. At 3.5 days postfertilization, embryos were pretreated in normoxic conditions ( $21 \%$ oxygen) with or without $10 \mu M$ nitrite, or hypoxic conditions ( $4 \%$ oxygen) with or without nitrite for $1 \mathrm{~h}$. The fin fold was then amputated posterior to the notochord, and embryos were allowed to recover in their treatment conditions (Fig. 8A).

Appropriate leukocyte recruitment is required to efficiently regenerate the fin fold (50). When we assayed the number of neutrophils that were recruited within $500 \mu \mathrm{m}$ of the amputation plane $16 \mathrm{~h}$ postamputation by using $T g(l y z: e G F P)^{k o O 2}$ embryos, hypoxia treatment decreased the total number of Lyzpositive neutrophils and addition of nitrite was able to restore the number of neutrophils back to normoxic levels (Fig. 8B, C), similar to the effect observed in the cardiac regeneration models (Fig. 7A, C and Supplementary Fig. S2C, D).

To determine whether the increased neutrophil recruitment was dependent on NO formation, larvae were treated with the NO scavenger, 2-4-carboxyphenyl-4,4,5,5-tetramethylimidazoline-1-oxyl-3-oxide (cPTIO) after amputation of the fin fold. cPTIO is a commonly used NO scavenger and has been shown to be effective in zebrafish larvae at a $10 \mu M$ dose (9). Treatment with hypoxia+cPTIO and hypoxia/nitrite+cPTIO abrogates the effect of nitrite and returns neutrophils levels back to hypoxia levels (Fig. 8B, C). Together, these data suggest that improved neutrophil recruitment by hypoxia/nitrite during fin regeneration is at least, in part, dependent on NO increased bioavailability derived from hypoxic nitrite reduction.

\section{Discussion}

As heart disease rates grow in an increasingly aging population, novel treatments to manage heart failure and myocardial infarction are sorely needed. A major therapeutic challenge is the design of strategies that reduce infarct size and improve cardiac repair after myocardial infarction. Although adult mammals lack the ability to regenerate the heart after injury, this capacity is preserved in the fetus and neonates and efforts to understand and reactivate this process are of great interest.

In this study, we found that physiological and therapeutic levels of nitrite increase the early recruitment of thrombocytes, neutrophils, and macrophages to the heart after amputation and cryoinjury in zebrafish, resulting in accelerated cardiomyocyte proliferation, angiogenesis, and cardiac regeneration.

In mammalian systems, nitrite has been shown to serve as a source of NO under hypoxic conditions via reduction by a variety of heme and molybdenum proteins, in particular $\mathrm{Hb}$, myoglobin, and xanthine oxidase $(44,64)$. Similar to mammals, fish NO synthases are oxygen dependent but the NO metabolites are maintained at similar levels in normoxia or hypoxia. These observations suggest that fish also exploit additional mechanisms to preserve NO homeostasis that are at play during hypoxia (20). Nitrite reduction to NO appears to be the main factor in these hypoxia-dependent mechanisms. The decrease in oxygen concentration increases the intracellular concentrations of nitrite, likely serving as a reservoir of NO for signaling (20).

Several studies have shown that exogenously administered nitrite limits tissue damage during ischemia-reperfusion events in mammalian models and it has been studied as a therapeutic agent for various cardiovascular diseases $(3,11$, 44). The mechanisms mediating this response are not completely understood, but they include effects on the inflammatory response and mitochondrial regulation via $\mathrm{NO}$ generation

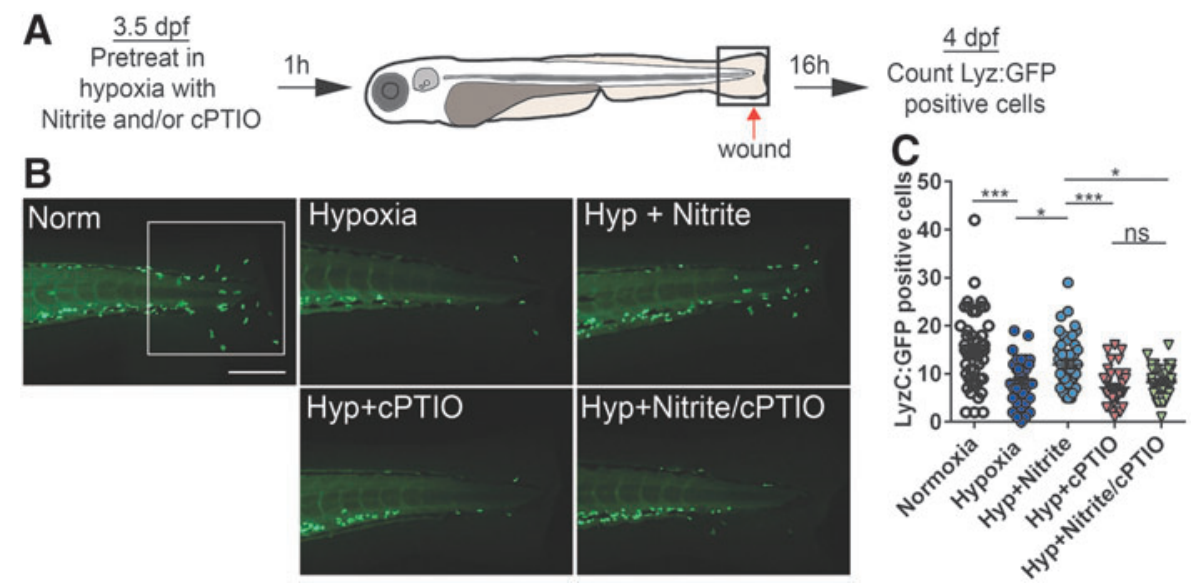

FIG. 8. Nitric oxide is required for the nitrite-induced increase in neutrophil recruitment in hypoxia after fin amputation. (A) Diagram of fin fold regeneration assay using zebrafish larval tail fins. Red arrow indicates amputation plane. Black box indicates the region within $500 \mu \mathrm{m}$ where Lyz-positive cells were quantified. (B) Representative images of $T g(l y z: G F P)^{k o O} 4 \mathrm{dpf}$ larvae 16 hpa treated with normoxia (norm), hypoxia, hypoxia $+10 \mu M$ nitrite, hypoxia $+10 \mu M \mathrm{cPTIO}$, and hypoxia $+10 \mu M$ nitrite and $10 \mu M$ cPTIO $(n=38-49)$. Green cells within the white box were quantified. Scale bar= $200 \mu \mathrm{m}$. (C). Quantification of Lyz-positive cells within $500 \mu \mathrm{m}$ of the amputation plane. One-Way ANOVA significance is $p<0.0001$. Tukey's multiple-comparison test; $* p<0.05, * * * p<0.001$. Data are expressed as mean \pm SEM. Each data point indicates an individual larva. cPTIO, 2-4-carboxyphenyl-4,4,5,5-tetramethylimidazoline-1-oxyl-3-oxide; dpf, days postfertilization; hpa, hours postamputation. 
and inhibition of mitochondrial complex I $(12,44,62)$. The function of nitrite and NO in fish metabolism has been explored only recently and never in a cardiac injury model (15, 20, 21, 59).

Here, we investigated the role of nitrite in heart regeneration by studying the fish's ability to recover after cardiac injury in water with $10 \mu M$ nitrite and low oxygen conditions. We found that the injury size is decreased in both amputation and cryoinjury models. This effect may be due to an early improvement in cardiomyocyte proliferation on treatment with nitrite in hypoxia along with improved neovascularization in the context of altered immune cell recruitment.

This suggests that hypoxia/nitrite is favorable during early events of the regenerative program, resulting in an improvement of the injury (52). These early events include a complex immune response that sets the stage for the injured heart to proceed toward a regenerative rather than scarring program, resulting in complete cardiac recovery $(10,23)$. Whether improved neovascularization is also a consequence of nitrite effect on the immune response preceding revascularization of the injury or whether it is determined directly by the effect of nitrite is a question that still needs to be explored.

Because of its role in cardiac repair, inflammation has long been considered a promising target for therapeutic interventions. Although numerous experimental studies demonstrate that influx of leukocytes and inflammatory mediators contribute to myocardial injury, clinical trials aiming at reducing these factors gave opposite results $(56,66,67)$. In models such as the salamander limb and zebrafish brain regeneration, inflammation is necessary and sufficient for enhancing regeneration $(18,35)$. In the zebrafish heart, the immune response is required to activate the regeneration program.

The regenerative capacity that is present in zebrafish and absent in medaka (Oryzias latipes) has been shown to be dependent on differences in immune cell dynamics between the two models. Small delays in macrophage recruitment can decrease neovascularization and heart regeneration, delay clearance of neutrophils, and promote scarring $(23,30,38$, 76). Hypoxia has been previously shown to favor cardiomyocyte proliferation in mammals and zebrafish heart regeneration $(29,49)$ but immediately after cardiac injury the early immune response seems to be negatively affected by hypoxia exposure.

Here, we found that hypoxia/nitrite can restore the early immune response to normal levels, potentially promoting hypoxia-driven cardiomyocyte proliferation. Immediately after cardiac injury, we found an increased number of thrombocytes in the scar in the presence of hypoxia/nitrite compared with hypoxia only. This effect precedes a higher level of neutrophil infiltration at 3-5 days after injury and it is followed by increased macrophages at 7 days during regeneration. This enhanced immune response could be induced by a larger scarring of the cardiac tissue in the hypoxic environment enriched with nitrite. However, at the same time, we measured a smaller injury and a decrease in erythrocytes in the clot, indicating that hypoxia/nitrite decreases scarring rather than promoting it. This is further supported by the subsequent improvement in cardiomyocyte proliferation and neovascularization.

Recently, important roles for MMPs during heart and fin regeneration have been demonstrated $(41,76)$. Expression of mmp $13 a$ is increased after cardiac cryoinjury in the zebrafish and inhibition of Mmp13a had a negative effect on heart regeneration through decreased recruitment of inflammatory cells to the site of injury (76). In addition, NO induces the expression and collagenase activity of MMP13, which regulates proliferation and migration of several cell types $(42,43$, $63,79)$. It is believed that Mmp13a acts to cleave the chemokine $\mathrm{Cxcl}$, which is highly chemoattractant to neutrophils after cleavage, supporting necessary immune cell infiltration to support cardiac regeneration (71).

In this study, we find that hypoxia/nitrite treatment increases the expression of mmpl3a within the injured heart and this increase correlates with improved immune cell recruitment to the injured area. It will be important to determine in future studies whether increased mmpl3a expression is reliant on increased NO bioavailability and whether this is responsible for the improved immune response in the hypoxia/nitrite-treated hearts.

We used the larval fin regeneration model to clarify the role of $\mathrm{NO}$ as a necessary intermediate of the nitritedependent effect on the immune response. In agreement with our cardiac data, the number of neutrophils migrating to the regenerating site is increased in hypoxia/nitrite, indicating that similar pathways are involved in the two regeneration models. The NO scavenger cPTIO alone did not alter neutrophil response in hypoxia but in combination with nitrite abolished the nitrite effect and decreased neutrophils similar to hypoxia levels. These data suggest that in hypoxic conditions, nitrite is the main source of NO production and that this NO generated is enough to impact the immune response.

It should be noted that hypoxia has been previously demonstrated to be pro-regenerative after cardiac injury in zebrafish (29). In this work, we do not observe an improvement in regeneration in either the heart or fin models after hypoxic treatment with the exception of cardiac injury size at $10 \mathrm{dpc}$.

Compared with the previously published work, here we focused on early time points and we used a different method to achieve hypoxia. Tissue hypoxia can be achieved by treatment with phenylhydrazine, an established method that causes anemia by inducing degradation of $\mathrm{Hb}$ and Myoglobin, effectively reducing oxygen delivery $(25,60)$. Importantly, in the presence of low oxygen in the heart, nitrite is the main source of NO made available by nitrite reduction through the action of globin proteins.

It is well characterized that at low oxygen tensions globins can act as nitrite reductases in mammals and fish $(8,62)$. Our results suggest that nitrite via reduction to NO impacts the immune response. Since our data point to nitrite reduction and involvement of the NO pathway, we excluded the use of the anemia model to achieve hypoxia because globins may play a fundamental role in zebrafish heart regeneration. Teasing apart the differences in results between our study and previously published work and their dependence on functional $\mathrm{Hb}$ and Myoglobin as well as other globins will be important for fully understanding the effects that low oxygen has on the regenerating myocardium. We foresee that a better understanding of the mechanisms of nitrite bioactivation during cardiac regeneration can help to delineate potential therapeutic interventions.

\section{Materials and Methods}

\section{Zebrafish strains and maintenance}

Adult zebrafish (D. rerio) were maintained according to standard protocols (75). Husbandry protocols and experiments 
were approved by the Institutional Animal Care and Use Committee (IACUC) at the University of Pittsburgh, which conforms to guidelines set by the NIH. The transgenic lines used, $T g(c d 41: e G F P)^{l a 2}$ (45), Tg(-2.4lyz:eGFP) ${ }^{k o 0}$ (33), Tg(gatala:DsRed $)^{s d 2}$ (69), Tg(flila:nEGFP) ${ }^{y 7}$ (58), Tg(kdrl: GFP $)^{l a 116}$ here referred to as $T g(f l k: G F P)(6)$, and Tg(mpeg 1: Dendra2) $)^{u w m 12}$ (77) have previously been described.

\section{Hypoxia and nitrite treatment}

Adult fish were maintained in hypoxic conditions by bubbling 5\% oxygen balanced nitrogen into the fish water for the duration of the experiment. Dissolved oxygen was measured by using a Mettle Toledo SevenGo Duo Pro dissolved oxygen meter (Fisher Scientific). Normoxic fish water contained 7-7.5 parts per million (ppm) oxygen, whereas 5\% oxygen-treated water contained $2.5-3.0 \mathrm{ppm}$ oxygen. To accurately measure baseline levels of nitrite in the system water, we regularly analyzed the water by using a Sievers NOA (model NOA 208i), which can detect nitrite concentrations with high sensitivity [as previously described, Corti et al. (8)]. Baseline nitrite concentrations ranged from undetectable to $0.14 \mu M$ (Fig. 1C). Sodium nitrite (563218; Sigma) was added to the water for a final concentration of $10 \mu M$, noting that water close to agricultural areas can reach $1 \mathrm{~m} M$ or higher, whereas natural water typically contains concentrations in the micromolar range (14). Adult fish were exposed to hypoxia, nitrite, or control conditions for 1 day before amputation or cryoinjury and for 3-7 days of recovery. Tanks were refreshed each day with fresh water with and without nitrite.

\section{Cardiac injury}

Adult fish were anesthetized in $0.168 \mathrm{~g} / \mathrm{L}$ ethyl 3aminobenzoate methanesulfonate salt (MS-222; Sigma) for 3-5 min. Zebrafish were placed ventral side up in a moistened sponge. A small incision was made to access the heart and either $20 \%$ of the ventricle was amputated as described (47, 52 ) or a stainless steel chilled cryoprobe was applied to the wall of the ventricle for $5 \mathrm{~s}$ as previously described for amputation and cryoinjury models, respectively (4). Fish were allowed to recover in normoxic conditions for $30 \mathrm{~min}$ to $1 \mathrm{~h}$ before being placed back into their respective hypoxic or normoxic condition, with and without nitrite.

\section{Histology, immunofluorescence, and imaging}

Hearts were collected from fish that had been euthanized in $0.168 \mathrm{~g} / \mathrm{L}$ ethyl 3-aminobenzoate methanesulfonate salt (MS222; Sigma) for $15 \mathrm{~min}$. After a brief wash in cold phosphate buffered saline (PBS), hearts were fixed either overnight at $4^{\circ} \mathrm{C}$ or for $2 \mathrm{~h}$ at room temperature in $4 \%$ paraformaldehyde. Hearts were rinsed twice in PBS, cryopreserved in a sucrose gradient, embedded in Surgipath Cryo-Gel (Leica), and frozen at $-80^{\circ} \mathrm{C}$. Twelve micrometer cryosections were collected on Superfrost Plus slides (Fisher), and consecutive sections were collected onto two different sets of slides. One set was stained with AFOG, and the other set was analyzed by immunofluorescence. AFOG staining was performed as described (52). The injured area was analyzed based on the largest middle sections of each heart, displaying the largest injury section. Three consecutive middle sections of the heart were analyzed by using Image (NIH), averaged, and used as a single data point. The size of the injury and of the remaining uninjured ventricle that was not stained for collagen and fibrin were measured and normalized to the total ventricular area (Fig. 1D). Because the variability in heart size correlates to fish length, the total ventricular area value was obtained by averaging four to six uninjured hearts extracted from fish of the same length as the fish used for each experiment. The primary antibodies used for immunostaining were anti $\alpha$-sarcomeric actin (A2172, 1:200; Sigma), anti-Mef2C (SC-313, 1:500; Santa Cruz Biotechnology), anti-Pcna (8825, 1:1000; Sigma), and anti-hypoxyprobe (NC01557961:50; Fisher Scientific). The secondary antibodies used for immunostaining (1:1000; Thermo Fisher) were goat-antirabbit Alexa Fluor 488 (A11008), goat-anti-rabbit Alexa Fluor Cy3 (A10520), goat-anti-mouse Alexa Fluor Cy3 (A10521), and goat-anti-mouse Alexa Fluor 488 (A11001). Slides were stained with DAPI (1:1000; Thermo Fisher), followed by treatment with Vectashield mounting medium (Vector Laboratories). Confocal images were taken with a Zeiss LSM 200 confocal microscope. Two-dimensional projections were generated from z-series ( $1 \mu \mathrm{m}$ steps). Lyz: GPF-positive cells, mpeg2:dendra1-positive cells, and Mef2C/ Pcna double-positive cells were manually counted over three separate consecutive sections, averaged, and normalized to the total area counted. $\operatorname{Tg}(c d 41: G F P)^{l a 2}, T g(\text { gatal:DsRed })^{s d 2}$, $T g(\text { flila: } n E G F P)^{y 7}$, and $T g(k d r l: G F P)^{l a l 16}$ fluorescence intensity was measured in $\mathrm{z}$-stacks from each heart considered over three consecutive sections by using ImageJ (NIH) and normalized to the injured area. Only hearts that had a clear injury located in the center sections of the heart were considered for each experiment.

\section{RNA isolation, complementary DNA synthesis, and $q R T-P C R$}

Ventricles from $3 \mathrm{dpa}$ fish treated with or without nitrite in normoxia or hypoxia were homogenized in $400 \mu \mathrm{L}$ TRIzol reagent (15596026; Thermo Fisher Scientific) by using a plastic microcentrifuge pestle. Eight hearts were pooled per RNA isolation by using the RNeasy Micro Kit (74034; Qiagen). One microgram of total RNA was used to generate complementary DNA (cDNA) by using Superscript II reverse transcriptase and an oligo (dT) primer (18064; Thermo Fisher Scientific) according to the manufacturer's protocol. cDNA was amplified in a quantitative polymerase chain reaction (qPCR) by using Taqman Universal PCR Master Mix (4364338; Thermo Fisher Scientific) and Taqman gene expression assay for mmp13a (Dr03438517_m1) and polr2d (Dr03095552_m1) in triplicate with appropriate nontemplate control reactions included. Expression of mmpl3a was normalized to polr $2 d$ expression by using the $\Delta \Delta \mathrm{C}_{\mathrm{T}}$ method, and the normoxic condition was used to calculate fold change.

\section{Hypoxyprobe}

Adult wild type zebrafish were intraperitoneally injected with $20 \mu \mathrm{L}$ of either $5 \mathrm{mg} / \mathrm{mL}$ (diluted in sterile PBS) hypoxyprobe (NC0155796; Fisher Scientific) or a PBS control for 3 days. Hearts were harvested, fixed in $4 \%$ paraformaldehyde, and processed for immunofluoresence as described earlier. 


\section{Fin fold amputation and analysis}

Three-day postfertilization embryos were anesthetized as described earlier for 2-3 min, and the fin fold was removed by using the tip of a 27-gauge needle just posterior to the notochord. Embryos were pretreated for $1 \mathrm{~h}$ before fin fold amputation and were maintained either at $4 \%$ oxygen in a hypoxic glove box or in an incubator with room air with or without $10 \mu M$ sodium nitrite in E3 media $(0.06 \mathrm{~g} / \mathrm{L}$ Instant Ocean) at $28.5^{\circ} \mathrm{C}$. At $4 \mathrm{dpa}$ (16 hours post fertilization), $\mathrm{Tg}(-2.4 \mathrm{lyz}$ : $e G F P)^{k o 02}$ embryos were imaged by using an MVX-10 MacroView microscope and DP71 camera (Olympus America). The number of LyzC:GFP-positive cells within $500 \mu \mathrm{m}$ of the fin fold were manually counted. Embryos were treated with $10 \mu M$ cPTIO (C221; Sigma) in $0.01 \%$ dimethyl sulfoxide (DMSO) beginning $1 \mathrm{~h}$ before fin amputation and kept for $16 \mathrm{~h}$.

\section{Statistics}

Data were analyzed by using a one-way ANOVA with Tukey's multiple comparisons to determine significance in cell counts and fluorescence intensity measurements when analyzing four treatment conditions by using GraphPad Prism software V6.0. Comparisons of only two treatment conditions were analyzed by Student's $t$-test. Each data point represents an individual animal/heart.

\section{Acknowledgments}

The authors thank D. Grainy and G. Blasko for zebrafish care and R. Handin for kindly providing the $\operatorname{tg}(c d 41: G F P)^{l a 2}$ fish line.

\section{Author Disclosure Statement}

J.T. is a co-inventor of pending patent applications and planned patents directed to the use of recombinant neuroglobin and heme-based molecules as antidotes for $\mathrm{CO}$ poisoning, which have recently been licensed by Globin Solutions, Inc. J.T. is Chief Scientific Officer and shareholder of Globin Solutions, Inc. M.T.G. is a co-inventor of pending patent applications and planned patents directed to the use of recombinant neuroglobin and heme-based molecules as antidotes for $\mathrm{CO}$ poisoning, which have recently been licensed by Globin Solutions, Inc. M.T.G. is a shareholder, advisor, and director in Globin Solutions, Inc. In addition, and unrelated to $\mathrm{CO}$ poisoning, M.T.G. is a co-inventor on patents directed to the use of nitrite salts in cardiovascular diseases, which have been licensed by United Therapeutics and Hope Pharmaceuticals, and is a co-investigator in a research collaboration with Bayer Pharmaceuticals to evaluate riociguat as a treatment for patients with sickle cell disease. M.T.G. has served as a consultant for Epizyme, Inc., Actelion Clinical Research, Inc., Acceleron Pharma, Inc., Catalyst Biosciences, Inc., Modus Therapeutics, and United Therapeutics Corporation. M.T.G. is also on Bayer HealthCare LLC's Heart and Vascular Disease Research Advisory Board. All other authors have no competing financial interests.

\section{Funding Information}

This work was supported by funding from Vitalant and the Hemophilia Center of Western Pennsylvania (to M.T.G.), Ri.MED Foundation (to P.C.) and by National Institutes of
Health Grants T32 HL110849 (to E.R.R.), R01 HL098032, P01 HL103455, T32 HL110849, and T32 HL007563 (to M.T.G.).

\section{Supplementary Material}

Supplementary Figure S1

Supplementary Figure S2

Supplementary Figure S3

\section{References}

1. Aurora AB, Porrello ER, Tan W, Mahmoud AI, Hill JA, Bassel-Duby R, Sadek HA, and Olson EN. Macrophages are required for neonatal heart regeneration. J Clin Invest 124: 1382-1392, 2014.

2. Broughton KM, Wang BJ, Firouzi F, Khalafalla F, Dimmeler S, Fernandez-Aviles F, and Sussman MA. Mechanisms of cardiac repair and regeneration. Circ Res 122: 1151-1163, 2018.

3. Bryan NS, Calvert JW, Elrod JW, Gundewar S, Ji SY, and Lefer DJ. Dietary nitrite supplementation protects against myocardial ischemia-reperfusion injury. Proc Natl Acad Sci U S A 104: 19144-19149, 2007.

4. Chablais F, Veit J, Rainer G, and Jazwinska A. The zebrafish heart regenerates after cryoinjury-induced myocardial infarction. BMC Dev Biol 11: 21, 2011.

5. Chen B and Frangogiannis NG. Immune cells in repair of the infarcted myocardium. Microcirculation 24: e12305, 2017.

6. Choi J, Dong L, Ahn J, Dao D, Hammerschmidt M, and Chen JN. FoxH1 negatively modulates flk1 gene expression and vascular formation in zebrafish. Dev Biol 304: 735-744, 2007.

7. Corti P, Ieraci M, and Tejero J. Characterization of zebrafish neuroglobin and cytoglobins 1 and 2: zebrafish cytoglobins provide insights into the transition from six-coordinate to five-coordinate globins. Nitric Oxide 53: 22-34, 2016.

8. Corti P, Xue J, Tejero J, Wajih N, Sun M, Stolz DB, Tsang M, Kim-Shapiro DB, and Gladwin MT. Globin X is a sixcoordinate globin that reduces nitrite to nitric oxide in fish red blood cells. Proc Natl Acad Sci U S A 113: 8538-8543, 2016.

9. Dahl Ejby Jensen L, Cao R, Hedlund EM, Soll I, Lundberg JO, Hauptmann G, Steffensen JF, and Cao Y. Nitric oxide permits hypoxia-induced lymphatic perfusion by controlling arterial-lymphatic conduits in zebrafish and glass catfish. Proc Natl Acad Sci U S A 106: 18408-18413, 2009.

10. de Preux Charles AS, Bise T, Baier F, Marro J, and Jazwinska A. Distinct effects of inflammation on preconditioning and regeneration of the adult zebrafish heart. Open Biol 6: 160102, 2016.

11. Dezfulian C, Raat N, Shiva S, and Gladwin MT. Role of the anion nitrite in ischemia-reperfusion cytoprotection and therapeutics. Cardiovasc Res 75: 327-338, 2007.

12. Duranski MR, Greer JJ, Dejam A, Jaganmohan S, Hogg N, Langston W, Patel RP, Yet SF, Wang X, Kevil CG, Gladwin MT, and Lefer DJ. Cytoprotective effects of nitrite during in vivo ischemia-reperfusion of the heart and liver. $J$ Clin Invest 115: 1232-1240, 2005.

13. Epelman S, Liu PP, and Mann DL. Role of innate and adaptive immune mechanisms in cardiac injury and repair. Nat Rev Immunol 15: 117-129, 2015.

14. Jensen FB. Uptake and effects of nitrite and nitrate in animals. In: Nitrogen Metabolism and Excretion, edited by Walsh WP and Wright P. Boca Raton: CRC Press, 1995, pp. 289-303.

15. Fago A, Parraga DG, Petersen EE, Kristensen N, Giouri L, and Jensen FB. A comparison of blood nitric oxide metab- 
olites and hemoglobin functional properties among diving mammals. Comp Biochem Physiol A Mol Integr Physiol 205: 35-40, 2017.

16. Frangogiannis NG. The immune system and cardiac repair. Pharmacol Res 58: 88-111, 2008.

17. Gladwin MT, Raat NJ, Shiva S, Dezfulian C, Hogg N, Kim-Shapiro DB, and Patel RP. Nitrite as a vascular endocrine nitric oxide reservoir that contributes to hypoxic signaling, cytoprotection, and vasodilation. Am J Physiol Heart Circ Physiol 291: H2026-H2035, 2006.

18. Godwin JW, Pinto AR, and Rosenthal NA. Macrophages are required for adult salamander limb regeneration. Proc Natl Acad Sci U S A 110: 9415-9420, 2013.

19. Gonzalez FM, Shiva S, Vincent PS, Ringwood LA, Hsu LY, Hon YY, Aletras AH, Cannon RO, 3rd, Gladwin MT, and Arai AE. Nitrite anion provides potent cytoprotective and antiapoptotic effects as adjunctive therapy to reperfusion for acute myocardial infarction. Circulation 117: 2986-2994, 2008.

20. Hansen MN and Jensen FB. Nitric oxide metabolites in goldfish under normoxic and hypoxic conditions. $J$ Exp Biol 213: 3593-3602, 2010.

21. Hansen MN, Lundberg JO, Filice M, Fago A, Christensen NM, and Jensen FB. The roles of tissue nitrate reductase activity and myoglobin in securing nitric oxide availability in deeply hypoxic crucian carp. J Exp Biol 219: 3875-3883, 2016.

22. Hendgen-Cotta UB, Merx MW, Shiva S, Schmitz J, Becher S, Klare JP, Steinhoff HJ, Goedecke A, Schrader J, Gladwin MT, Kelm M, and Rassaf T. Nitrite reductase activity of myoglobin regulates respiration and cellular viability in myocardial ischemia-reperfusion injury. Proc Natl Acad Sci U S A 105: 10256-10261, 2008.

23. Huang WC, Yang CC, Chen IH, Liu YM, Chang SJ, and Chuang YJ. Treatment of glucocorticoids inhibited early immune responses and impaired cardiac repair in adult zebrafish. PLoS One 8: e66613, 2013.

24. Huang Z, Shiva S, Kim-Shapiro DB, Patel RP, Ringwood LA, Irby CE, Huang KT, Ho C, Hogg N, Schechter AN, and Gladwin MT. Enzymatic function of hemoglobin as a nitrite reductase that produces NO under allosteric control. J Clin Invest 115: 2099-2107, 2005.

25. Jain SK and Subrahmanyam D. On the mechanism of phenylhydrazine-induced hemolytic anemia. Biochem Biophys Res Commun 82: 1320-1324, 1978.

26. Jayaraman T, Tejero J, Chen BB, Blood AB, Frizzell S, Shapiro C, Tiso M, Hood BL, Wang X, Zhao X, Conrads TP, Mallampalli RK, and Gladwin MT. 14-3-3 binding and phosphorylation of neuroglobin during hypoxia modulate sixto-five heme pocket coordination and rate of nitrite reduction to nitric oxide. J Biol Chem 286: 42679-42689, 2011.

27. Jensen FB. Nitric oxide formation from nitrite in zebrafish. J Exp Biol 210: 3387-3394, 2007.

28. Jopling C, Sleep E, Raya M, Marti M, Raya A, and Izpisua Belmonte JC. Zebrafish heart regeneration occurs by cardiomyocyte dedifferentiation and proliferation. Nature 464: 606-609, 2010.

29. Jopling C, Sune G, Faucherre A, Fabregat C, and Izpisua Belmonte JC. Hypoxia induces myocardial regeneration in zebrafish. Circulation 126: 3017-3027, 2012.

30. Karra R, Knecht AK, Kikuchi K, and Poss KD. Myocardial NF-kappaB activation is essential for zebrafish heart regeneration. Proc Natl Acad Sci U S A 112: 13255-13260, 2015.

31. Khandekar G, Kim S, and Jagadeeswaran P. Zebrafish thrombocytes: functions and origins. Adv Hematol 2012: 857058, 2012.
32. Kikuchi K, Holdway JE, Werdich AA, Anderson RM, Fang Y, Egnaczyk GF, Evans T, Macrae CA, Stainier DY, and Poss KD. Primary contribution to zebrafish heart regeneration by gata4(+) cardiomyocytes. Nature 464: 601-605, 2010.

33. Kitaguchi T, Kawakami K, and Kawahara A. Transcriptional regulation of a myeloid-lineage specific gene lysozyme C during zebrafish myelopoiesis. Mech Dev 126: 314-323, 2009.

34. Kumar D, Branch BG, Pattillo CB, Hood J, Thoma S, Simpson S, Illum S, Arora N, Chidlow JH, Jr., Langston W, Teng X, Lefer DJ, Patel RP, and Kevil CG. Chronic sodium nitrite therapy augments ischemia-induced angiogenesis and arteriogenesis. Proc Natl Acad Sci U S A 105: 75407545, 2008.

35. Kyritsis N, Kizil C, Zocher S, Kroehne V, Kaslin J, Freudenreich D, Iltzsche A, and Brand $\mathrm{M}$. Acute inflammation initiates the regenerative response in the adult zebrafish brain. Science 338: 1353-1356, 2012.

36. Laflamme MA and Murry CE. Heart regeneration. Nature 473: 326-335, 2011.

37. Lahnsteiner F. The sensitivity and reproducibility of the zebrafish (Danio rerio) embryo test for the screening of waste water quality and for testing the toxicity of chemicals. Altern Lab Anim 36: 299-311, 2008.

38. Lai SL, Marin-Juez R, Moura PL, Kuenne C, Lai JKH, Tsedeke AT, Guenther S, Looso M, and Stainier DY. Reciprocal analyses in zebrafish and medaka reveal that harnessing the immune response promotes cardiac regeneration. Elife 6: e25605, 2017.

39. Li H, Samouilov A, Liu X, and Zweier JL. Characterization of the magnitude and kinetics of xanthine oxidasecatalyzed nitrate reduction: evaluation of its role in nitrite and nitric oxide generation in anoxic tissues. Biochemistry 42: 1150-1159, 2003.

40. Li HT, Hemann C, Abdelghany TM, El-Mahdy MA, and Zweier JL. Characterization of the mechanism and magnitude of cytoglobin-mediated nitrite reduction and nitric oxide generation under anaerobic conditions. $\mathrm{J}$ Biol Chem 287: 36623-36633, 2012.

41. Li L, Zhang J, and Akimenko MA. Inhibition of mmp13a during zebrafish fin regeneration disrupts fin growth, osteoblasts differentiation, and Laminin organization. Dev Dyn $1-12,2019$.

42. Lizarbe TR, Garcia-Rama C, Tarin C, Saura M, Calvo E, Lopez JA, Lopez-Otin C, Folgueras AR, Lamas S, and Zaragoza C. Nitric oxide elicits functional MMP-13 protein-tyrosine nitration during wound repair. FASEB $J$ 22: 3207-3215, 2008.

43. Lopez-Rivera E, Lizarbe TR, Martinez-Moreno M, LopezNovoa JM, Rodriguez-Barbero A, Rodrigo J, Fernandez AP, Alvarez-Barrientos A, Lamas S, and Zaragoza C. Matrix metalloproteinase 13 mediates nitric oxide activation of endothelial cell migration. Proc Natl Acad Sci U S A 102: 3685-3690, 2005.

44. Lundberg JO, Weitzberg E, and Gladwin MT. The nitratenitrite-nitric oxide pathway in physiology and therapeutics. Nat Rev Drug Discov 7: 156-167, 2008.

45. Ma D, Zhang J, Lin HF, Italiano J, and Handin RI. The identification and characterization of zebrafish hematopoietic stem cells. Blood 118: 289-297, 2011.

46. Marin-Juez R, Marass M, Gauvrit S, Rossi A, Lai SL, Materna SC, Black BL, and Stainier DY. Fast revascularization of the injured area is essential to support zebrafish 
heart regeneration. Proc Natl Acad Sci U S A 113: 1123711242, 2016.

47. Missinato MA, Saydmohammed M, Zuppo DA, Rao KS, Opie GW, Kuhn B, and Tsang M. Dusp6 attenuates Ras/ MAPK signaling to limit zebrafish heart regeneration. Development 145: dev157206, 2018.

48. Morales RA and Allende ML. Peripheral macrophages promote tissue regeneration in zebrafish by fine-tuning the inflammatory response. Front Immunol 10: 253, 2019.

49. Nakada Y, Canseco DC, Thet S, Abdisalaam S, Asaithamby A, Santos CX, Shah AM, Zhang H, Faber JE, Kinter MT, Szweda LI, Xing C, Hu Z, Deberardinis RJ, Schiattarella G, Hill JA, Oz O, Lu Z, Zhang CC, Kimura $\mathrm{W}$, and Sadek HA. Hypoxia induces heart regeneration in adult mice. Nature 541: 222-227, 2017.

50. Nguyen-Chi M, Laplace-Builhe B, Travnickova J, LuzCrawford P, Tejedor G, Lutfalla G, Kissa K, Jorgensen C, and Djouad F. TNF signaling and macrophages govern fin regeneration in zebrafish larvae. Cell Death Dis 8: e2979, 2017.

51. Nurden AT. Platelets inflammation and tissue regeneration. Thromb Haemost 105(Suppl 1): S13-S33, 2011.

52. Poss KD, Wilson LG, and Keating MT. Heart regeneration in zebrafish. Science 298: 2188-2190, 2002.

53. Qian F, Zhen F, Ong C, Jin SW, Meng Soo H, Stainier DY, Lin S, Peng J, and Wen Z. Microarray analysis of zebrafish cloche mutant using amplified cDNA and identification of potential downstream target genes. Dev Dyn 233: 11631172, 2005.

54. Rassaf T, Flogel U, Drexhage C, Hendgen-Cotta U, Kelm $M$, and Schrader J. Nitrite reductase function of deoxymyoglobin: oxygen sensor and regulator of cardiac energetics and function. Circ Res 100: 1749-1754, 2007.

55. Reeder BJ and Ukeri J. Strong modulation of nitrite reductase activity of cytoglobin by disulfide bond oxidation: implications for nitric oxide homeostasis. Nitric Oxide 72: 16-23, 2018.

56. Roberts R, DeMello V, and Sobel BE. Deleterious effects of methylprednisolone in patients with myocardial infarction. Circulation 53: I204-I206, 1976.

57. Rodriguez J, Maloney RE, Rassaf T, Bryan NS, and Feelisch M. Chemical nature of nitric oxide storage forms in rat vascular tissue. Proc Natl Acad Sci U S A 100: 336-341, 2003.

58. Roman BL, Pham VN, Lawson ND, Kulik M, Childs S, Lekven AC, Garrity DM, Moon RT, Fishman MC, Lechleider RJ, and Weinstein BM. Disruption of acvrl1 increases endothelial cell number in zebrafish cranial vessels. Development 129: 3009-3019, 2002.

59. Sandvik GK, Nilsson GE, and Jensen FB. Dramatic increase of nitrite levels in hearts of anoxia-exposed crucian carp supporting a role in cardioprotection. Am J Physiol Regul Integr Comp Physiol 302: R468-R477, 2012.

60. Shetlar MD and Hill HA. Reactions of hemoglobin with phenylhydrazine: a review of selected aspects. Environ Health Perspect 64: 265-281, 1985.

61. Shiva S and Gladwin MT. Nitrite mediates cytoprotection after ischemia/reperfusion by modulating mitochondrial function. Basic Res Cardiol 104: 113-119, 2009.

62. Shiva S, Sack MN, Greer JJ, Duranski M, Ringwood LA, Burwell L, Wang X, MacArthur PH, Shoja A, Raghavachari N, Calvert JW, Brookes PS, Lefer DJ, and Gladwin MT. Nitrite augments tolerance to ischemia/reperfusion injury via the modulation of mitochondrial electron transfer. J Exp Med 204: 2089-2102, 2007.

63. Tarin C, Gomez M, Calvo E, Lopez JA, and Zaragoza C. Endothelial nitric oxide deficiency reduces MMP-13mediated cleavage of ICAM-1 in vascular endothelium: a role in atherosclerosis. Arterioscler Thromb Vasc Biol 29: 27-32, 2009.

64. Tejero J, Shiva S, and Gladwin MT. Sources of vascular nitric oxide and reactive oxygen species and their regulation. Physiol Rev 99: 311-379, 2019.

65. Tidball JG and Villalta SA. Regulatory interactions between muscle and the immune system during muscle regeneration. Am J Physiol Regul Integr Comp Physiol 298: R1173-R1187, 2010.

66. Timmers L, Pasterkamp G, de Hoog VC, Arslan F, Appelman Y, and de Kleijn DP. The innate immune response in reperfused myocardium. Cardiovasc Res 94: 276-283, 2012.

67. Timmers L, Sluijter JP, van Keulen JK, Hoefer IE, Nederhoff MG, Goumans MJ, Doevendans PA, van Echteld CJ, Joles JA, Quax PH, Piek JJ, Pasterkamp G, and de Kleijn DP. Toll-like receptor 4 mediates maladaptive left ventricular remodeling and impairs cardiac function after myocardial infarction. Circ Res 102: 257-264, 2008.

68. Tiso M, Tejero J, Basu S, Azarov I, Wang XD, Simplaceanu V, Frizzell S, Jayaraman T, Geary L, Shapiro C, Ho C, Shiva S, Kim-Shapiro DB, and Gladwin MT. Human neuroglobin functions as a redox regulated nitrite reductase. J Biol Chem 286: 18277-18289, 2011.

69. Traver D, Paw BH, Poss KD, Penberthy WT, Lin S, and Zon LI. Transplantation and in vivo imaging of multilineage engraftment in zebrafish bloodless mutants. Nat Immunol 4: 1238-1246, 2003.

70. Tzahor $\mathrm{E}$ and Poss KD. Cardiac regeneration strategies: staying young at heart. Science 356: 1035-1039, 2017.

71. Van den Steen PE, Proost P, Wuyts A, Van Damme J, and Opdenakker G. Neutrophil gelatinase B potentiates interleukin- 8 tenfold by aminoterminal processing, whereas it degrades CTAP-III, PF-4, and GRO-alpha and leaves RANTES and MCP-2 intact. Blood 96: 2673-2681, 2000.

72. van der Meer DL, van den Thillart GE, Witte F, de Bakker MA, Besser J, Richardson MK, Spaink HP, Leito JT, and Bagowski CP. Gene expression profiling of the long-term adaptive response to hypoxia in the gills of adult zebrafish. Am J Physiol Regul Integr Comp Physiol 289: R1512R1519, 2005.

73. Verollet C, Charriere GM, Labrousse A, Cougoule C, Le Cabec V, and Maridonneau-Parini I. Extracellular proteolysis in macrophage migration: losing grip for a breakthrough. Eur J Immunol 41: 2805-2813, 2011.

74. Voslarova E, Pištěková V, and Svobodova Z. Nitrite toxicity to Danio rerio: effects of fish age and chloride concentrains. Acta Vet Brno 75: 107-113, 2006.

75. Westerfield M. The Zebrafish Book. A Guide for the Laboratory Use of Zebrafish (Danio rerio), 3rd ed. Eugene, OR: University of Oregon Press, 1995, p. 385.

76. Xu S, Webb SE, Lau TCK, and Cheng SH. Matrix metalloproteinases (MMPs) mediate leukocyte recruitment during the inflammatory phase of zebrafish heart regeneration. Sci Rep 8: 7199, 2018.

77. Yoo SK and Huttenlocher A. Spatiotemporal photolabeling of neutrophil trafficking during inflammation in live zebrafish. J Leukoc Biol 89: 661-667, 2011. 
78. Yoong S, O'Connell B, Soanes A, Crowhurst MO, Lieschke GJ, and Ward AC. Characterization of the zebrafish matrix metalloproteinase 9 gene and its developmental expression pattern. Gene Expr Patterns 7: 39-46, 2007.

79. Zaragoza C, Balbin M, Lopez-Otin C, and Lamas S. Nitric oxide regulates matrix metalloprotease-13 expression and activity in endothelium. Kidney Int 61: 804-808, 2002.

80. Zuppo DA and Tsang M. Fusion heals the broken-hearted. Ann Transl Med 6: S21, 2018.

Address correspondence to: Dr. Mark T. Gladwin Department of Medicine Heart, Lung, Blood, and Vascular Medicine Institute University of Pittsburgh Pittsburgh, PA 15213

E-mail: gladwinmt@upmc.edu

Dr. Paola Corti Department of Medicine Heart, Lung, Blood, and Vascular Medicine Institute University of Pittsburgh Pittsburgh, PA 15213

E-mail: pac47@pitt.edu
Date of first submission to ARS Central, November 7, 2018; date of final revised submission, November 4, 2019; date of acceptance, November 13, 2019.

\section{Abbreviations Used}

$$
\begin{aligned}
& \text { AFOG }= \text { Acid Fuchsin Orange } \mathrm{G} \\
& \text { ANOVA }=\text { analysis of variance } \\
& \mathrm{cDNA}=\text { complementary DNA } \\
& \mathrm{cPTIO}= 2-4 \text {-carboxyphenyl-4,4,5,5- } \\
& \quad \text { tetramethylimidazoline-1- } \\
& \text { oxyl-3-oxide } \\
& \mathrm{dpa}= \text { days post-amputation } \\
& \mathrm{dpc}= \text { days post-cryoinjury } \\
& \mathrm{Hb}= \text { hemoglobin } \\
& \mathrm{Mb}= \text { myoglobin } \\
& \mathrm{mef} 2 \mathrm{C}= \text { myocyte enhancer factor } 2 \mathrm{c} \\
& \mathrm{MMPs}= \text { matrix metalloproteinases } \\
& \mathrm{NO}= \text { nitric oxide } \\
& \mathrm{NOA}= \text { nitric oxide analyzer } \\
& \mathrm{PBS}= \text { phosphate buffered saline } \\
& \mathrm{Pcna}= \text { proliferating cell nuclear antigen } \\
& \mathrm{ppm}= \text { parts per million } \\
& \mathrm{qRT}-\mathrm{PCR}= \text { quantitative reverse transcription } \\
& \text { polymerase chain reaction }
\end{aligned}
$$

